\title{
Venn Symmetry and Prime Numbers: A Seductive Proof Revisited
}

Stan Wagon, Macalester College, St. Paul, MN 55105 (wagon@macalester.edu) [corresponding author]

Peter Webb, School of Mathematics, University of Minnesota, Minneapolis, MN 55455

(webb@math.umn.edu)

When he was an undergraduate at Swarthmore College in 1960, David W. Henderson [Hen] proved an important and surprising theorem about Venn diagrams.

Theorem (Henderson). If a symmetric $n$-Venn diagram exists, then $n$ is prime.

An $n$-Venn diagram is a Venn diagram on $n$ sets, which is defined to be a collection of $n$ simple closed curves (Jordan curves) $C_{1}, C_{2}, \ldots, C_{n}$ in the plane such that any two intersect in finitely many points and each of the $2^{n}$ sets of the form $\bigcap C_{i}^{\epsilon_{i}}$ is nonempty and connected, where $\epsilon_{i}$ is one of "interior" or "exterior". Thus the Venn regions are all bounded except for the region exterior to all curves; the finite intersection property means that each bounded region is the interior of a Jordan curve. See [RW] for much more information on Venn diagrams. An $n$-Venn diagram is symmetric if each curve $C_{i}$ is $\rho^{i}\left(C_{1}\right)$, where $\rho$ is a rotation of order $n$ about some center (we use $O$ for the fixed point of rotation $\rho$ ).

We often use Boolean representations: $111 \ldots 1$ represents $F$, the full intersection of all the interiors, $000 \ldots 0$ is the empty set, and $100 \ldots 0$ represents the set of points interior to $C_{1}$ and exterior to the others. In a symmetric Venn diagram, rotation of a region by $\rho$ corresponds to a rightward shift of the Boolean string.

The universally familiar three-circle Venn diagram is symmetric, as is the one on two sets using two circles. For about 40 years a major open question was whether symmetric $n$-Venn diagrams exist for all prime $n$. Henderson found one for $n=5$ and also (unpublished) for $n=7$. Much later, Hamburger [Ham] settled the case of 11, which was quite complicated, and then in 2004 Griggs, Killian, and Savage [GKS] found an approach that works for all primes. So we now have the strikingly beautiful theorem that a symmetric $n$-Venn diagram exists if and only if $n$ is prime.

But there is a small problem: Henderson's proof, which appears to be very simple, has a gap. Here is the proof from [Hen].

Suppose $1 \leq k \leq n-1$. Since a symmetric $n$-Venn diagram is symmetric with respect to a rotation of $2 \pi / n$, the regions corresponding to the $k$-subsets of $\{1,2, \ldots, n\}$ must come in groups of size $n$. Therefore $n$ divides $\left(\begin{array}{l}n \\ k\end{array}\right)$. But it is well known and easy to prove (see [RSW]) that the only $n$ for which this is true for the specified $k$-values are the primes. 
This is a very seductive argument. The primeness arises in such a cute way that one wants it to be true. Thus the proof has been repeated in many papers in the decades since it was first published. Yet there are problems. First, the proof does not mention the connectedness of the Venn regions. Without connectedness the result is false; see Figure 1 (from [G]), which shows a disconnected symmetric diagram for $n=4$. More important, the reasoning that the Venn regions come in bunches of $n$ is insufficient, since it could perhaps happen that a Venn region $R$ coincides with one of its rotated copies, thus disrupting the count. Certainly $R$ cannot equal $\rho(R)$, because a Boolean string with $k 1$ s must have a 0 , and therefore one rightward shift yields a different string, and therefore a region disjoint from $R$. However, some patterns are invariant under two rotations: the string 1010 is unchanged by two rightward shifts. This phenomenon would invalidate the proof, so to fix it one must show that in a symmetric $n$-Venn diagram, $\rho^{i}(R)$ cannot equal $R$ if $1 \leq i \leq n-1$.

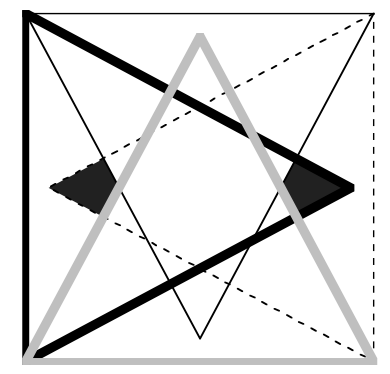

Figure 1. Rotating an equilateral triangle leads to a symmetric diagram that represents all 16 combinations of the four sets. But the set that represents a combination is not necessarily connected (as in the shaded case).

To fill the gap we present a lemma that can be used either to fix Henderson's proof or to create a more direct proof with no reference to binomial coefficients. We need the Jordan curve theorem: Every simple closed curve $J$ in the plane divides the complement of $J$ into two connected open sets, with $J$ being the boundary of each.

Lemma. Let $J$ be a simple closed curve invariant under a nonidentity rotation $\rho$ about center $O$. Then $O$ is in the interior of $J$.

Proof. Let $S$ be the exterior of $J$. Let $x$ be a point on $J$ that is farthest from $O$ and let $C$ denote the circle centered at $O$ and passing through $x$; therefore $J$ is contained in $C$ and its interior. Note that $J$ travels from $x$ to $\rho(x)$ and then back to $x$, thus splitting into two paths, which we call $J_{1}$ and $J_{2}$. Suppose $O$ is not in the interior of $J$. Then either $O \in S$ or $O$ is on $J$. In the first case, let $z=O$. In the second case, when $O$ is on $J$ - on $J_{1}$, say - there is a small disk $D$ about $O$ that is disjoint from $J_{2}$. Such a disk must contain a point $z \in S$. In either case, there is an unbounded path that starts at $z$ and is contained in $S$. Let $P$ be the truncation of such a path as soon as it reaches circle $C$, so that $P$ goes from $z$ to a point $y$ on $C$ and in $S$.

Let $B$ be a circle with center $O$ and radius larger than that of $C$. Form the simple closed curve $K$ that starts at $x$, follows path $J_{2}$ to $\rho(x)$, goes radially outward to $B$, follows an arc of $B$ to the point radially aligned with $x$, and then goes radially into $x$. Of the two possible $B$-arcs, use the one that places $z$, indeed all of $D$, in the interior of $K$. The use of the larger circle guarantees that $K^{\prime}$ s return trip to $x$ misses $J$. Using the fact that $y$, being in $S$, cannot rotate to a point on $J$, we can rotate path $P$ to $\rho^{i}(P)=Q$ so that $y$ moves to $u$, a point on $C$ whose radial extension to infinity does not intersect $K$ (Fig. 2). Symmetry implies that $Q$ is contained in $S$. Because $\rho^{i}(z)$ is in disk $D$, it lies in the interior of $K$. But $u$ is in the exterior of $K$. Thus $Q$ 
connects the interior of $K$ to its exterior, which means that $Q$ intersects $K$, a contradiction as a common point would be on or inside $C$ and so would be both in $S$ and on $J$.

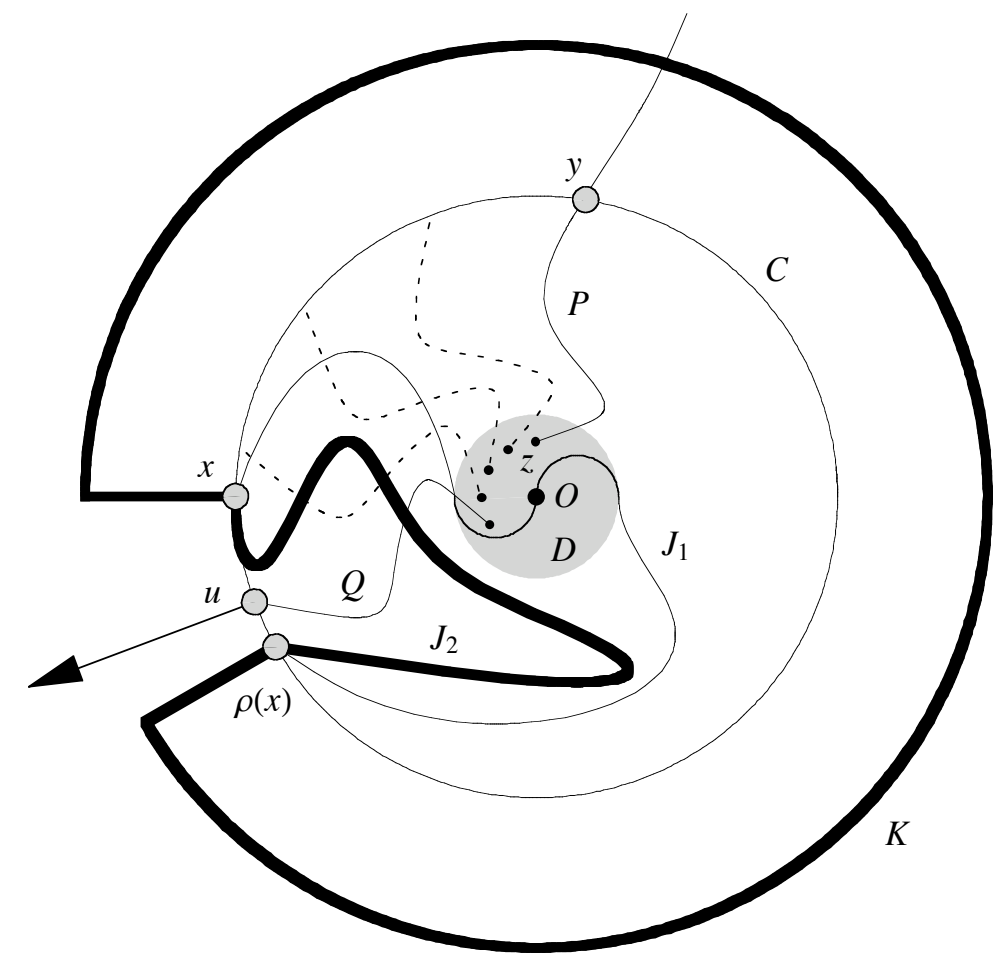

Figure 2. A schematic representation of the situation where $O$ is on $J$. The point $z$ is in the exterior of $J$ and near $O$. Then one forms a path $P$ from $z$ to $C$ and rotates the path to a path $Q$ that connects the interior of $K$ to its exterior.

Note the useful consequence of the lemma: In a symmetric Venn diagram $O$ lies in $F$, the full intersection region. This is because $F$, and therefore its bounding curve, are invariant under the rotation. The lemma sheds light on Henderson's proof as follows. Let $R$ be a Venn region as in that proof. Then if $1 \leq i \leq n-1$, it cannot happen that $\rho^{i}(R)=R$, since the lemma, applied to the boundary of $R$, would place $O$ in $R$. Since $O$ must also be in $F$, this is a contradiction. Therefore $n$ does indeed divide $\left(\begin{array}{l}n \\ k\end{array}\right)$ whenever $1 \leq k \leq n-1$, as claimed. Binomial coefficients are not really relevant, and we can eliminate them entirely by the following direct proof.

Proof of Theorem. Suppose the assertion is false, so that there is a composite number $n$ with proper divisor $b$ and a Jordan curve $V$ such that, for a rotation $\rho$ of order $n$, the curves $\rho^{i}(V), i=0, \ldots, n-1$, form an $n$-Venn diagram. Let $R$ be the region with Boolean representation $100100 \ldots 100$, with $b$ bits in each block (here $b=3$ ). Then both $R$ and $\rho(R)$ are invariant under $\rho^{b}$ and so the lemma places $O$ in each of them. This is a contradiction because the two sets are distinct Venn regions $(\rho(R)$ 's Boolean representation begins $01 \ldots$ ).

We acknowledge email exchanges with Branko Grünbaum and David Henderson in the course of which alternative approaches were proposed. These involved the use of winding numbers and also the reduction of the given Venn diagram to one in which each curve is piecewise linear. Such approaches work, but ours is based on a lemma that makes no reference to Venn diagrams, and so provides a purely geometric proof of a geometric fact. 


\section{REFERENCES}

[GKS] J. Griggs, C. E. Killian, and C. D. Savage, Venn diagrams and symmetric chain decompositions in the Boolean lattice, Electronic Journal of Combinatorics, 11 (2004), \#R2.

[G] B. Grünbaum, Venn diagrams and independent families of sets, Mathematics Magazine, 48 (1975) 12-23.

[Ham] P. Hamburger, Doodles and doilies, non-simple symmetric Venn diagrams, Discrete Mathematics, 257 (2002) 423-39.

[Hen] D. W. Henderson, Venn diagrams for more than four classes, this Monthly, 70 (1963) 424-26.

[RSW] F. Ruskey, C. Savage, and S. Wagon, The search for simple symmetric Venn diagrams, Notices of the American Mathematical Society, 53 (2006) 1304-12.

[RW] F. Ruskey and M. Weston, A survey of Venn diagrams, Electronic Journal of Combinatorics, 4 DS5 (1997). 